\title{
From the writable web to the global editability
}

\author{
Angelo Di lorio \\ University of Bologna \\ Mura Anteo Zamboni 7 \\ 40100 Bologna (Italy) \\ +390512094871 \\ diiorio@cs.unibo.it
}

\author{
Fabio Vitali \\ University of Bologna \\ Mura Anteo Zamboni 7 \\ 40100 Bologna (Italy) \\ +390512094872 \\ fabio@cs.unibo.it
}

\begin{abstract}
The technical and competence requirements for writing content on the web is still one of the major factors in widening the gap between authors and readers. Although tools that support an easy approach to web writing, such as blogs and wikis, are becoming increasingly important and mainstream, they still lack in terms of layout and typographical sophistication, and, most importantly, only allow local editing (on the pages that are stored by the application itself). In this paper we re-propose an old paradigm for writing content on the net, directly derived from the Xanadu vision by Ted Nelson: global editability foresees that all documents on the web can be accessed for editing and modified on line, very much as in a global wiki. Global eidtability needs to address a number of issues, most notably correct support for intellectual property and legal issues, before it can be accepted as an idea. We provide some further considerations on that, as well as describe our own architecture and implementation of such a system, called IsaWiki.
\end{abstract}

\section{Categories and Subject Descriptors}

H.5.1 [Information Interfaces and Presentation] Hypertext/Hypermedia

H.5.3 [Information Interfaces and Presentation] Web-based Interaction

\section{General Terms \\ Design}

\section{Keywords}

Web authoring, global editability, collaboration, customization, data collection.

\section{INTRODUCTION}

Today surfing the web is simple and fast: an internet connection, a common browser and few skills allow users to search and read a huge amount of information for their work and personal interests. On the contrary, writing the web is not so straightforward and easy but it still requires expertise and proper tools and writepermissions. Yet, a lot of different solutions exist for the creation of web pages, more or less simple: from the advanced editors used by the professionals to the simple editors for unaware users, from the content management systems to the wikis and so on. On the surface, web authoring may seem completely addressed but a lot of issues still need to be solved [11], first of all the strong asymmetry between the roles of the author and the reader.
While a reader can easily read information, in fact, an author needs to master specific technologies and tools and have write permissions on a server. Thus, the first step towards the overlapping of these two roles needs to address the simplification of the editing process.

Originally the web browsers could also be used to create new pages and links, but after the advent and the success of Mosaic, these editing capabilities were increasingly dismissed. Recently the W3C introduced Amaya [1], a browser/editor allowing users to directly modify the web pages during the navigation, within the browser. Such approach has some evident benefits for the users, since they do not need additional skills and tools for editing, and they do not have to switch between different applications.

Improvement of our daily web experience, which we call "web augmentation" does not simply happen with a simplification of the editing process, though: the most important innovation we strive for is the ability of performing personal versions of other authors' pages and customizing all web content regardless of their ownerships and write permissions. Although web users today can easily read web pages they still cannot add content, annotate and customize other peoples' materials during the navigation.

Such a vision has been deeply studied in the field of open hypermedia in the 90s: many systems were proposed to allow readers to add links and annotations to a wide range of existing documents, integrating original content and external interventions [18][2]. Unfortunately the World Wide Web moved towards a different direction and many advanced hypertextual functionalities has been neglected in its design, such as private and public links, personalization, reuse of contents, on-line editing and so on[3]. However several proposals addressed the integration of hypermedia concepts into the World Wide Web, such as DLS [6], Webvise [15], and Arakne [5].

This integration could not be considered the definitive step towards a sharable and global publishing environment. Indeed, our model can be considered Xanadu [30], a universal environment where everyone can access, read, re-use, modify and comment any material of other users, tailoring it to his/her own purpose. Xanadu never came to exist but its principles and features are still topical. In this paper we describe a system called IsaWiki [12] that aims to transform the Web into a universal knowledge base from which all users can draw information and ideas, profiting from the contributions of the others as in Xanadu. The key feature of the IsaWiki system is in fact the global editability of the pages.

At first glance, a global editing environment could seem a shared workspace where all pages can be modified by all users in a chaotic and uncontrolled way. This is a scenario that can be 
avoided and that we want to avoid. On the contrary we think that customization, collection and reuse of other peoples' materials can be the right moves towards an augmented web publishing environment, if and only if they are performed in a controlled and safe way.

Global editing in our view does not mean hacking, i.e. modifying and substituting content in web pages regardless of ownerships and write permissions, but rather creating new resources and personalize the existing ones, drawing contents and ideas from a knowledge-base shared by all the web users.

This paper just focuses the attention on global editability as a new paradigm for the creation of web authoring applications. In particular, in section 2 we will discuss the benefits of such an approach comparing it with related proposals and works. In section 3 some technical and non-technical issues about global editability are discussed. Finally, section 4 describes the implementation details of IsaWiki, a system developed at the University of Bologna that takes these ideas to implementation.

\section{GLOBAL EDITABILITY}

Several different approaches have been developed towards the overlapping of the roles of the reader and the author in the current World Wide Web. The common goal of these solutions is just the recovery of the advanced hypertextual functionalities that the web has lost with time and, in particular, the transformation of all the users from passive readers into active writers. However, any existing proposal addresses a specific problem, proposes targeted solutions and meets only partial requirements of a complete editing environment. Global editability is a new paradigm for designing web authoring applications that aims to merge all the existing approaches into a single, universal and more powerful one. Indeed, it is an old authoring model adapted to the current World Wide Web. We just define "global editability" (GE) the possibility for any web user to modify, customize and re-use any web content in a controlled and safe way. We think that the operations already provided by different augmented web authoring systems can be further improved and integrated each other, just through GE.

In the literature four different approaches for an augmented web authoring can be identified: writable web [4][9], external layered contributions[22][8], harvestable data collection [33][27] and collaborative editing [30][24].

\subsection{Writable Web}

The term "writable web" indicates the possibility for the users of writing web content with the same skills and tools used to read them. Recently two proposals are increasingly gaining importance towards this goal: weblogs and wikis. Weblogs [4] are tools for fast editing (mostly based on web forms) and publishing of personal diaries, addressed to individuals and small communities. Wikis[9] are collaborative tools for shared writing and browsing, allowing every reader to access and edit any page of the site during navigation through simple web forms and a very intuitive text-based syntax for special typographical effects. They are characterized by simple interfaces and an open editing philosophy that encourages participation. On the other hand, wikis are limited in that the overall esthetics of the created pages is inherently poor, users have to learn yet another text-based editing language, and there is no fine-grained control over single contributions beyond a basic diff tool. The most important feature of these solutions is just the integration between the browsing and the editing processes: the same skills and tools, in fact, allow users either to read or to write content with few efforts.

Wikis and weblogs, with a few exceptions, are based on HTML forms. A further step towards a writable web is the possibility for the users of modifying content within the browser and the page being used, though a WYSIWYG editor. This editing is just called in-place editing since the whole structure and content of the page remain in the main browser window, during the editing. Such approach offers some evident benefits to the users: no additional tool and skill is required, no switching between different applications can disorientate users and the editing can be easily performed contextually with the browsing.

The integration of surfing and editing the web cannot be limited to the simplicity in performing such operations, though: not only local documents have to be editable but this property has to be extended to all web pages. In a sense, wikis and weblogs are "local": pages are designed and inserted in a special structure to be edited. Although all web users can edit them during the browsing, the editability is limited to the local resources. On the contrary, GE entitles any user to modify, customize and re-use any content during the browsing. Not only the web designed for this purpose is writable but the whole web is a global knowledge base shared among all users.

\subsection{External layered contributions}

We call "external layered contributions" the external annotations and links that users can add to the web documents. Several projects, in fact, have been developed in order to provide users the possibility of annotating, criticizing and commenting on external resources, regardless of their locations, access permissions and ownership [5][20]. All these systems are based on the same principle: any user can annotate any page, since these interventions are stored externally and, whenever the page is accessed, they are inserted on-the-fly into the original one (that remains unmodified on the origin server). In particular, such systems were proposed for external links and annotations.

Arakne[5] and iMarkup[20] are two clear examples of external annotation systems, allowing users to add external notes and comments to any web page. Recently the W3C introduced Annotea[22], a framework based on RDF for defining and distributing annotations on web pages. The same browser/editor Amaya, described in the introduction, supports the Annotea protocol for the creation and distribution of external annotations.

Similarly, the XLink standard gives users the possibility of creating links externally to the resources they connect. XLinkProxy[8] is a system for external linking of web pages, implemented as a non-transparent HTTP proxy: whenever a user requests a page, if links exist in a external linkbase, they are added on-the-fly into the original document before displaying it.

Yet, external annotations and links meet only some tasks desirable in a really sharable editing environment, since users can only add a single layer of annotations onto the original documents. The annotations are simply added to the original content: there no deep intervention on the actual content of the page, no deletion, no change in the overall structure and so on. Instead, in a system based on the GE approach, we wish all users to freely customize and reuse content without limits of size, users and permissions. Personal modifications can change over the time, different variants can be created and contributions from different sources and authors can converge into any document. Customizations and external changes, in fact, cannot be limited to an extra layer of 
data integrated into the original page, but they have to be considered as complex and unpredictable interventions that have to be able to occur without particular constraints.

\subsection{Harvestable data collection}

None of the documents we write can be considered an independent and isolated unit: in any document a lot of explicit and implicit links to existing material can be found, as well as citations and references to related works, fragments of content directly imported from other pages and so on. New documents can be obtained by combining existing information (collected from different resources) and additional contents added by the author. We just call "harvestable data collection" the possibility of collating and retrieving fragments of content from different web pages, in order to re-use them in new pages.

No tools exist now to perform these actions directly. The tools required to implement this process are separate and the process is far from simple and straightforward in the World Wide Web: rather, it requires a lot of expertise.

One of the possible outcomes of such a process are personal anthologies of web content about a specific topic. Such anthologies would be composed of the reported articles (or parts thereof) as well as comments, annotations and summaries written by the users. Today, except from some specific tools [33][27] described further on, the process is based on explicit operations performed manually through individual tools: bookmarking, copy\&paste and word-processing $(B C W)$ :

- Bookmarks are used to store and retrieve references to the interesting pages,

- $\quad$ copy\&paste is used to select, extract and insert existing fragments into a new document and

- word-processing is used to merge personal comments and adjust pasted and new text in the final document.

The BCW approach has some evident limits and drawbacks. First of all, it requires some expertise from the users, since different skills and tools are required and no direct support for the sequence of operations is provided by any of them. Secondly, switching between applications can be perceived as a nuisance and possibly even an obstacle by the users which are forced to divide their attention between different tasks and tools [39].

Finally, this method is unsatisfactory considering how the references and links to the original documents are managed: when a user merges fragments form different sources into the same document, in fact, all the references to the original sources are lost (and need to be explicitly inserted by the author) and new contributions are indistinguishable from the original ones. Thus, all the connections and relationships between the documents and their original sources are difficult to restore. Furthermore, there is no control over the reuse of other people material since all users can copy web content and paste them into their documents without the document recording any hint of their origin. Many thorny issues exist related to the intellectual propriety management and we have dedicated section 3.2 of this paper to them.

The BCW approach can be improved by GE, since the same actions could be performed in a straightforward more powerful way. In this case, GE stands for the possibility of collating fragments of all the accessed pages and re-using them in the creation of new materials and archiving references to the original content. The key-aspect is just the capability of segmenting pages into individually manageable assets of content, identifying any contribution within the document, and connecting all the occurrences of the same material.

New scenarios can derive from such approach: all the imported fragments could be highlighted and identifiable, so that readers could go back to the original sources, view metadata about the fragments, view information about the original author and so on, if they so wished. These fragments would be directly copied from the original source to the new document and information and links would be automatically added by the system.

In the hypertext literature advanced solutions were proposed in order to connect occurrences of the same information: disappeared but not forgotten have been the proposals for hot links, warm links and transclusions. Warm and hot links were defined [26][7] as "live" connections between two end-points through which data can move from one document to the other: whenever one of the two end-points is modified the other is automatically updated. From a technical point of view, warm and hot links are actual copies of data enriched with references to the original content.

Transclusions [28] are pointers to the original data. Transclusions are the core mechanism of the Xanadu system [30], an ambitious idea by Ted Nelson from which the same Web took inspiration. Xanadu is a universal environment where everyone can access, read, re-use, modify and comment any material of other users, tailoring it to his/her own purpose. A Xanadu document is not stored as a whole block but as a list of references to fragments combined in the final document, still connected to the original ones and automatically updated. Actually there exists a unique instance of any fragment that is included (or better transcluded) in all places that use it.

More recently, Hunter Gatherer [33] is a very good implementation of a web data collection tool. It is a browserintegrated solution that allows users to collate information within web pages and organize them in structured and navigable collections. Hunter Gatherer is based on a special copy\&paste operation similar to the Xanadu transclusions: the user surfs a page, selects a fragment and copies it to his current collection. The collection appears as a list of clickable links through which the user can move to the original document or display the fragment in a floating window. Hunter Gatherer manages the information collections as structured sets of fragments and links but does not allow users to re-use content in new documents.

MRS [27] is a management reporting system that allows users to harvest content form external sources and compound them into other documents. The application was designed to support report management in a firm where the roles are hierarchically structured: managers should be able use content from the reports produced by their subordinates as basis for their new reports. A server-side application manages the collections and a plug-in for Microsoft Word allows users to create and edit composite documents assembling results from the others' reports.

MRS was just designed for a closed environment where a set of users can modify a set of documents stored on a local network. We think that the web data collection process must involve all the web pages as Hunter Gatherer does and, at the same time, it must be a complete editor for composite documents such as MRS. GE just merges these two requirements so that the huge amount of information retrievable from the web can be easily collected and 
imported in new documents, keeping references to the original sources of information.

\subsection{Collaborative editing and emergent collaboration}

Collaborative editing, i.e. the possibility of producing documents in collaboration and actually changing the resource acting collaboration, has been deeply studied by researchers and professionals and a lot of advanced groupware applications have been proposed [23].

We think that collaborative editing does not need a new application but a deep transformation of the Web into a universal environment where all users can modify, customize and collaborate on other peoples' materials, once again GE.

The existing collaborative editing tools, in fact, are designed to fully support collaboration among users belonging to the same group. On the contrary, GE provides for extending collaboration not only to a predefined set of users but potentially to all web users that can customize and collaborate on all web resources.

This vision moves off some projects proposed many years ago, such as Xanadu and Rhythm [24]. Rhythm took inspiration from Xanadu and provided a lot of similar functionalities, including free linking, annotation, inclusion and document modification to all users and a complete scalability relatively to the number of users, documents and links.

The GE approach can be useful and suitable for the current World Wide Web too. In this new scenario a web user publishes a page about a specific topic and sets which users are entitled to create official versions of the same page. Whenever an authorized user modifies the page (through an editor integrated in the browser) this version becomes the official one, otherwise a personal variant is created. All the users that do not belong to the team of the official authors, can annotate, comment and revise personal variants of the page for their own purposes and develop their own tangled trees of personal modifications.

Since versions and variants are composed of original fragments, personal interventions and imported materials clearly distinguishable from each other, web pages are modified in a safe way with regards to intellectual property: on the one hand, all interventions are stored externally and do not interfere with the original content; on the other hand it is always possible to trace who edited, when edited and what was edited.

Any user can also propose his/her personal modifications to the owner of a document that would examine, approve and make them official. In [25] we defined this new form of collaboration an "emergent collaboration", which is not any form of pre-defined cooperation (between users belonging to the same group) but a spontaneous and unforeseen interaction of strangers. The group of emergent collaborators can even be extended to the whole set of the web users, so that the publishing environment could be considered a huge set of potential collaborators.

A positive aspect of this "emergent collaboration" is as basic as evident: unexpected and unpredictable interactions can spontaneously come out so that a public document can be enriched with unexpected and new contributions. The authors can improve their public pages exploiting suggestions and content from his/her emergent collaborators; in the same way all users can improve their personal documents drawing ideas and content from the others. Since personal documents can be easily transformed or partially imported into the public ones, all web pages can forage each other.

Another outcome is the improvement of the quality of the documents. Such approach can be likened to the open-source philosophy [32], according to which several revisers and developers contribute towards the same task and everyone share his/her skills and knowledge with the others. Discussing the pros and cons of open-source, especially with regards to information content, is out of the scope of this paper but such a pluralistic and democratic approach can be naturally cast into this context. Furthermore, while the process of the production of software requires an engineered and methodical approach, a more dynamic and unforeseen behavior can be equally useful and suitable in the case of multi-authored documents.

Finally, the users belonging to the spontaneous and unforeseen group of emergent collaborators do not necessarily belong to the same community and they do not have the same skills and background. Allowing them to spontaneously collaborate on web pages can be a vehicle for sharing and merging variegated wealth of experience, knowledge and education. The WWW is not only a huge and cold pool of documents, but it could become a pool of human resources and individuals joined in spontaneous communities. Thus, the collaborative production could be a vehicle for the birth and development of communities around specific topics as it has already happened around several of the most successful wikis.

Wikis [9] are the closest proposals to the GE approach. As we said, the main limit of a wiki is that a wiki can only edit local resources while such a process should be performed on just any resource of the web. Even those wikis that give the possibility of modifying wiki pages to all users, including unregistered ones, provide editability remains only to the local resources.

A particularly interesting proposal among the wikis is JotSpot [21]. JotSpot is a wiki providing all the basic functionalities in a simpler way than the average wiki (for instance, it provides a WYSIWYG editor). The pages are not unstructured text but the users can integrate advanced elements such as calendars, forms, tables from submitted data, polls and emails and so on. External web content can be integrated too, such as results from a searchengine or news from a newsgroup. JotSpot partially extends the wiki concept over the whole Web: external materials can be collected and integrated in the pages but the editability still involves only the local resources. On the contrary the GE paradigm provides users for modifying and collaborating on all web pages, regardless of their write permissions, ownerships and locations.

\section{SOME TECHNICAL AND NON- TECHNICAL ISSUES IN GLOBAL EDITABILITY}

Complex technical and non-technical issues about the feasibility of GE exist and need to be deeply investigated. In this section we will just underline some of them as we are foreseeing them, describing the open problems and proposing our solutions. In the subsequent section we will describe IsaWiki a system being developed at the University of Bologna that takes these solutions to implementation.

In particular, five issues about $\mathrm{GE}$ need to be clarified:

- Technical feasibility of the GE 
- Intellectual propriety management

- Assets, content and layout management

- Versioning

- Scalability

\subsection{Technical feasibility of the global editability}

Today the WWW is increasingly becoming a place for supplying services and showing products and initiatives, rather than remaining a hypertextual web of connected content. Web pages are often complicated and full of graphical elements, since their main goal is catching users' attention and winning an audience over the competition. Thus, is it possible to revolutionize the current WWW architecture and twist behaviors and practices of millions of users towards $G E$ ? The answer is obviously negative.

GE cannot involve uprooting the current WWW structure and forcing an inadmissible revolution on a system so stable and widespread, but rather building on the current WWW an infrastructure to provide users all the required functionalities for publishing and collaboration. In particular it does not involve to:

- Suggest new protocols, a new architecture or new standards and languages, instead of the existing ones.

- Destroy the current WWW with all the services, e-commerce tools, graphical elements, lovely sites, collaborative tools, and so on.

On the contrary, it aims to:

- Exploit the existing technologies and protocol towards the creation of a new environment that will not revolutionize the current one but will coexist and augment the WWW.

- Create an environment addressed only to the interested users, without interfering with the navigation and the publishing processes of the rest of the WWW.

Nonetheless, not everyone would be happy if the web becomes a universally writable environment, and not all circumstances and uses allow or require this. For instance, in [11] two possible publishing philosophies are identified:

- Restricted publishing: where only authorized authors use the innovative tools to create content that is subsequently officially published, and where particularly important are process management features and easier life for authors.

- Open publishing: where every reader is authorized to use the innovative tools and contribute to existing content.

In the first case (for instance, a corporate CMS) GE is not a required feature but a functionality to avoid; on the other hand in the second it could be very useful as well as expected.

While some pages are naturally suitable for customization and reuse, many others are not: for example, could it really be interesting to customize a name list? Why a user should be interested in the personalization of a bus timetable? The GE model provides the technical support for customization and re-use of all web documents and let users choose when and where modifying the pages, according to their preferences and needs.

Not all web users are interested in GE and many of them do not like to be "disturbed" during the navigation. For the GE approach to work, it needs to be similar to the opt-in philosophy of "good" email marketing that makes sure that only interested users are contacted [31]. GE needs to be designed as a service for registered users: subscribers surf on personal variants of the pages and can access all the contributions, while the others surf on the original pages as created by the original author.

\subsection{Intellectual propriety management}

All web users can modify and customize all web content: were intellectual propriety management neglected in our design? GE simply by-passes the problem: instead of preventing malicious and illegal reuse of others' people materials, it provides an infrastructure for monitoring who and when modifies content. Thus, users will be able to control intellectual propriety and copyrights.

Currently the problem of copyright are dealt with by Digital Rights Management systems, tools to control and limit the use of digital materials. After the explosion of digital music and filesharing on the Internet, DRM has gained increasingly importance up to become a hot and crucial topic for all content providers. In [14] DRMS are divided into two generations: the first-generation relies on security and encryption to solve copyright problems, that is, lock the content and limit its distribution to only authorized users, the second-generation defines languages and standards to describe the information assets and define right holders relationships and protection rules. Different proposals were published based on the same schema: modeling the entities to be protected, identifying and describing these assets and expressing the rights statements over them. DOIs[34] are names used to identify and exchange assets and intellectual properties, ODRL[19] is a language to express rights information over content.

A particularly interesting approach, from which our same vision of GE has drawn inspiration, is transcopyright [28] as proposed by Ted Nelson for Xanadu. The concept of transcopyright is inseparable from the concept of transclusions. A Xanadu document is a virtual list of fragments that are "transcluded" from the original source. The owner of each fragment can decide if the other users have to pay for the transclusion of the materials. Ideally any single byte of information can be priced so that a user customizing or re-using others' materials has to pay for it.

Proposing the transcopyright in the current WWW is certainly not admissible for two relevant reasons: first, Xanadu exploited a complex and all-in-all outdated schema numbering; second, the WWW is so rooted in its current form that it is impossible to impose users to pay for the reuse and customization of others' materials. However the main principle underlying transcopyright, that is, the total traceability of the interventions, can be adapted for the current WWW too.

The GE idea does not prevent users to copy protected materials but requires that traces all the usages of content are registered and stored. Paradoxically this freedom should be up being a limit for malicious and illegal practices since all changes can be monitored and verified [40]. The distinction between original (and protected) content and personal variants could thus become a barrier against malicious interventions and at the same time a basis for the support of systems for innovative copyright control and management. The first required step, intrinsic in the GE vision, is the complete traceability of the modifications and the external anchoring; mechanisms to control, to prevent or to subject to payment the usage of some materials can be easily implemented on top of these data. 


\subsection{Assets, content and layout management}

The GE model presupposes an advanced and fine-grained management of the documents' fragments: each document should be divisible into a set of assets storable, manageable and reusable in independent way.

Xanadu addressed this issue exploiting a specific numbering schema called tumblers [29] and many other projects pre-web used their own addressing schemas. The situation has changed with the advent of the HTML, since mechanisms based on offsets and bytes counting are now unsatisfactory. Two standards, XPath and XPointer, have been introduced just to provide addressing and identification mechanisms for any fragment of an XML document but, even if we managed to convert all current HTML pages into XHTML, such mechanisms would not be enough to solve the problem of addressing. Two issues are relevant: the presence of multiple data formats and the fusion (and indeed confusion) of content and layout in the WWW documents.

Most web pages are coded in HTML but a lot of other data formats are readable through a web browser, such as PDF, Doc, SVG, XML and so on. In order to modify these documents and reuse significant fragments it is necessary to convert them from a data format to another and vice versa. The same operation is required if we aim at comparing two different documents in different formats or including fragments from an external resource. Obviously proper tools can be used to view and modify content in a specific format, but this solution does not allow different sources to be really integrated each other: each fragment is a black box only readable with a specific tool rather than a really convertible and reusable unit of content.

By implementing a superior standard model [13], that is, an intermediate generic data format with features from both source and destination formats, the conversion of each formats to and from this one becomes possible. A basic assumption lies beneath this idea: that any document in any data format has a representation in the generic standard model that is capture at least the real content of the document, that is, the most relevant part for reuse.

In fact, users customizing web pages are probably interested in the actual content of the pages, rather than their presentational aspects. Probably when including fragments into a personal anthology or into a new document they need to end up with a layout and the graphical elements that are appropriate to the new document, and not the source (as it would be in most cases for $\mathrm{BCW}$ tasks). On the other hand, the same assets management modules within the system need to handle content units rather than layout information. Thus, the generic data format needs to be designed to capture the actual semantic information within the document. This conversion must be done with the smallest loss of information and must extract (in case, storing externally) all the details about the layout and the style.

This content and layout extraction is particularly interesting when applied to HTML pages. In the last years, in fact, graphical elements are increasingly gaining importance in the design and coding of the web pages; the result is that several pages are composed of images, logos, footers, animations and many other advanced graphical elements heavily intermixed with the actual content of the page. Thus, it is very hard to distinguish the actual content from the presentational aspects: a tool for the extraction of the layout information from a web page and the identification of the role of each fragment is required in order to implement the GE paradigm [37].

The generic data format neglects all the graphical aspects, since the system breaks down a document in two distinguished components, the content and the layout. The advantages of a clear distinction between information and presentation are already known: first of all, the authors can deal only with the real content of a document and not with its decoration and style; secondly, the content production could be fast and easy [35]. This distinction allows users to organize, analyze, correct and modify the pages according to their semantic information too. Finally the same content could be represented with various layouts and displayed by different devices [16].

In the GE scenario extracting content from a document is on the one hand a useful extra feature but, on the other hand, a necessary step towards the integration of different sources of information: in fact, the users can edit documents sharing fragments from any source and re-flowing them in any layout, without limit of data formats and layouts.

\subsection{Versioning}

In the field of hypermedia the advantages of versioning have already been described [36] [38]: historical revision, security and explorative production, distributed and asynchronous collaboration, emergent forms of collaboration, workflow support, efficiency and scalability.

In the GE model versioning surely is an extra feature providing services and information to the users but, also, it is a necessary requirement. An advanced versioning and diff engine is necessary to trace the contributions of the different authors of different sources, to outline differences between two documents, to restore old changes, to revise and accept modifications, to differentiate all the personal interventions and so on.

The simplest approach to providing these features is to implement a session-based mechanism for versioning and external anchoring. It allows users to modify HTML pages through an WYSIWYG editor, during the browsing. After an editing session, a new version of the document is sent to the central server the user registered to: if the user belongs to the list of official authors a new official version of the document is created and becomes immediately available, otherwise a new item is added to the tree of the author's personal variants. This also and most importantly allows the the user to customize web pages that are not necessarily stored on the central servers. Delta-based versioning would be an additional feature whereby each version/variant is not entirely saved on the server but only as the differences between two subsequent versions, according to a forward-delta-based schema. Intermediate versions would be built on demand according to the readers' requests.

Since any document on any server needs to be versioned, any fragment of any version (or personal variant) can be univocally identified by a URL, indicating the document name and version, and an XPath (or XPointer) indicating the fragment.

Of course, although all the official versions and the personal variants are automatically versioned on the central server, the original web pages (still stored on the origin server) are not. How can these documents be integrated in the whole versioning system?

The problem does not occur in the case of page customization, since whenever a personal variant is created the system saves the 
entire document (in case of transformation of a set of versions into a chain of deltas, the first variant is entirely saved on the server yet) and so, even if the original page is modified, all the required information are still available on the central server. Starting from the first one all the subsequent variants are wholly versioned by the system.

For anthologies, where some fragments are imported from ordinary web pages, the problem needs to be addressed. If a user includes a fragment from a web page that will be later modified on the origin-server, all the connections and information about this page will be soon groundless. To solve this problem without losing efficiency and scalability, solutions based on caching mechanisms, data replication and consistency checks need to be addressed.

\subsection{Scalability}

Another important question concerns scalability. Highly used web pages could be modified and customized by several hundreds or thousands of users so that a huge number of personalized versions could have to be managed: how can a system based on GE scale? We think that GE could be fully realized by a distributed and decentralized environment where many servers live together providing services for many users. Each user initially registers himself to a single server that will support him during the navigation and the editing. All the operations regarding the documents personalization, editing and storage are managed by the server the user have registered to. Thus, the critical aspect is the number of the registered users (and consequently the amount of personal variants) on a particular server rather than the total number of existing versions or the number of users modifying the same web page. So the system could easily scale by increasing the number of the servers, by replicating resources and by exploiting techniques related to the distributed systems.

In conclusion, in this section we have identified some relevant requirements of the GE model. To summarize, a publishing environment based on such approach has to:

- Coexist with the current WWW, without revolutionizing its architecture and protocols

- Not interfere with the navigation of uninterested users

- Not cover uninterested areas or circumstances where it is not suitable

- Monitor all the interventions being able to trace who edited, when edited and what was edited

- Be extensible with copyright management tools, protocols and languages

- Manage content assets with advanced mechanisms based on a clear distinction between content and layout

- Be independent from the data formats

- Support advanced versioning

- Be completly scalable

\section{ISAWIKI}

The requirements above are the cornerstones of the design of IsaWiki, a publishing environment which takes GE to implementation, under development at the University of Bologna. IsaWiki is a distributed system providing services to registered users. It is a simple client-side architecture based on the HTTP protocol. An IsaWiki server is a PHP application running on a common web server that provides services and advanced functionalities of document management. An IsaWiki client is a plug-in for some common web browsers that monitors the user's navigation, sends requests to the IsaWiki server and allows users to edit the pages. Thus, the system naturally coexists with the architecture, the protocols and the languages and tools of the current Web.

A web user interested in the IsaWiki services has to simply install the plug-in and register himself to an IsaWiki server. From now on, user's editing and surfing will be monitored and supported by the system. What do the terms "monitored and supported" mean? Let us go into the implementation details of the system, in order to explain.

Some interface elements (sidebars, toolbars, menus and buttons) can be added to the both major browsers of current generation, Internet Explorer and Mozilla, to provide users advanced functionalities during the browsing within the accessed page. For instance, a sidebar is a module residing with the browser that can monitor the URL loaded in the main window, associate an action to each event, start autonomous HTTP connections and wholly modify the DOM of the page in the main window. The IsaWiki client is just a sidebar exploiting such functionalities and having a full control on the current page in the browser.

The following figure shows the IsaWiki interface during the browsing:

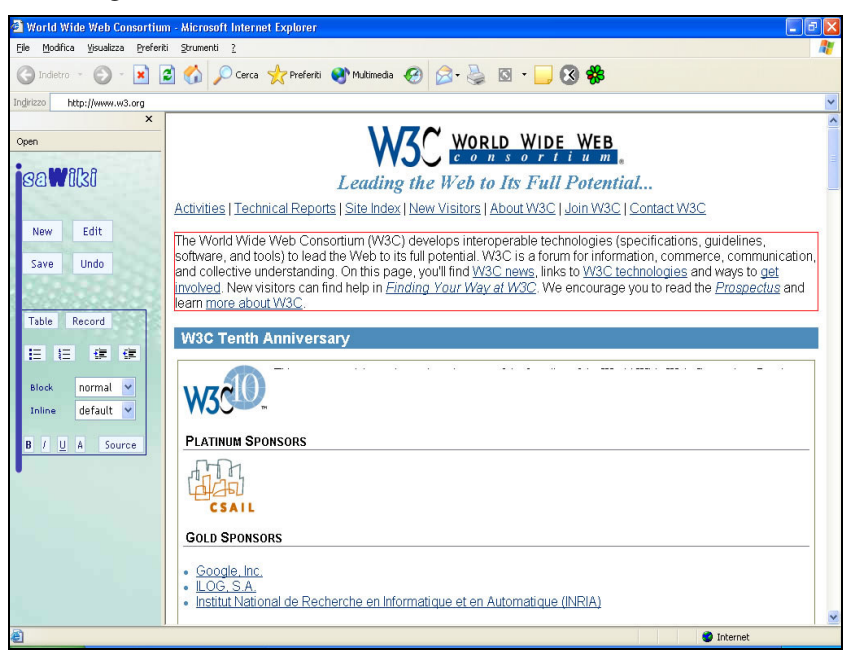

Figure 1. The IsaWiki sidebar.

Let us briefly describe what happens whenever a user surfs a web page: the sidebar catches the event URL-change in the navigation bar, verifies (through a HTTP request to the preset server) if a personal variant of the current page exists. In case, a personalized version is displayed, in stead of the original page from the originserver.

A versions/variants list is always displayed of documents to let the user access every individual previous state of the document. The original copy is always selectable, to let the user access the document on its real origin server. If the document on the origin server has changed since the first modification of the customized version, the current version of the document is also shown in the list as an autonomous version. Furthermore, if the page has been already handled by the system (this means that it is not a common web page but an official version of an IsaWiki document or a personal variant and so the system has already segmented the page in identifiable content assets) a list of selectable different data 
formats which the page can be converted is available too. Currently IsaWiki supports conversion from and to HTML, XML, Wiki, TeX, PDF (partially) and .Doc.

Clicking on the edit button the user can customize the current page, directly during the browsing, through a WYSIWYG editor. The figure 2 shows a zoom on the editor buttons:

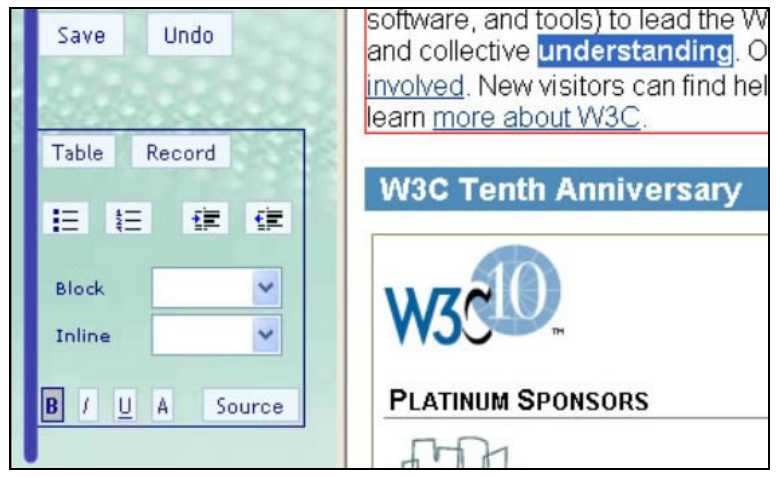

Figure 2. The IsaWiki Editor

Before analyzing the editor's features, we need to underline a significant aspect already discussed in the section 3.3: web pages should not be customized keeping undistinguishable content and layout but it should be easier and more useful to segment a page into individually manageable assets of information. However today the web pages are far from being so clearly structured: firstly, they are not well-formed in XHTML but not even conformable to the HTML specifications since the browsers are able to digest and display quite everything; secondly the actual content of a page is heavily intermixed with graphics and layouts, so that, it is very hard to extract information from graphics, logos, footers, headers and so on. For this reason, we have integrated in IsaWiki a web pages' structural analysis tool, called elISA [37], able to identify the real content of a page, by studying structures, patterns and regularities in the HTML code. elISA is actually implemented as a rule-based transformation engine that, once applied to HTML pages, adds appropriate attributes and other markup to the original document to indicate the role of each fragment. Conditions and criterions to determine these roles are expressed through declarative rules, in a special XML syntax. A meta-stylesheet is applied to the rules file and generates the actual XSLT that, in turn, outputs the final result. Of course, being based on a heuristic approach, these techniques cannot be perfect and universal but, extending and polishing the rules, much better results can be achieved.

Although elISA is able to gather the role of other page elements, in IsaWiki it is simply used to determine the areas containing the actual content of the page. Only these areas can be modified by the user, while the other areas remain visible in the browser window for contextualization and orientation of the user. Internet Explorer and Mozilla have recently introduced some properties of the page elements, such as contentEditable in Internet Explorer and designMode in Mozilla, just to provide the in-place editing of the content within the actual page that hosts the original content, with the same styles and layouts. Thus, elISA decides which fragments of the page are the actual content and labels these fragments with proper attributes, after the IsaWiki editor allows user to only edit these zones.

Within the editable regions, which are emphasized with bordered boxes, any content and structure can be freely modified, deleted or added and the DOM is consequently and immediately updated according to these modifications. As expected, the commands of the editor reflect the generic data format model. Few elements compose our generic data format: blocks (e.g. paragraphs) containing text and inline elements (e.g. bold and italics), tables containing individual cells, collections of records (e.g. a drawing composed of many simple graphical elements in a vector-based graphic language), lists, and little else. The main idea is that each concept can be expressed with different constructions in the different data formats and can be associated to different styles but it conceptually remains the same thing. For instance, a paragraph with style 'important' in MS Word, a fragment $<\mathrm{p}$ class='important' $>$ An important paragraph $\langle/ p>$ in HTML, and the fragment <important>An important paragraph $</$ important $>$ in XML are all equivalent, and can be easily transformed back and forth from one format to the others.

The user can create content elements from scratch (in this case, a form to insert meta-information is displayed too) or modify the role and the properties of a selected fragment of the area. In the figure 2, the user was emphasizing a word (in bold) but he should be able to invent and assign a new customized element.

The editor produces a page wholly conformable to the IsaWiki generic data format, even if does not limit the possibilities and the capabilities of the user. Thus, the final page could be easily segmented by the server into content assets and used for a GEbased environment. However the most web pages are obviously not natively conformable to the IsaWiki data format, so the first step performed by the editor is just the transformation into this format of the HTML code of the page. Obviously this transformation can lose some information but it has been just designed to minimize this loss and to keep any relevant content.

After the editing session, the sidebar sends the modified document to the IsaWiki server adding a few metadata such as the title of the document, the access list for reading and writing, the modification time, the author and so on. This information is useful to manage, version and organize the documents on the server but we plan in the near future to extend them towards a more complete metadata management.

Server-side, IsaWiki provides all the services to support the functionalities in the client-side interface and, in particular, handles different versions, fragments, layouts and formats of the documents. Let us go a little back: a user can modify the HTML pages through the IsaWiki editor, the documents in PDF or DOC directly uploading new versions on the server, the documents in XML or Wiki though a simple text editor. In fact, a user can also ask to edit the page invoking a specific script on the server that will extract the content of the page, convert it into the generic data format, and place it within an HTML form to be displayed to the user. So, also old browsers can be used for editing, as evidence of the fact that the IsaWiki servers can used by any web client, with few more efforts.

IsaWiki implements a session-based versioning and external anchoring. Whenever the user sends (or copies) a modified document to the server, in fact, according to whether he has write permissions or not, an official version or a personal variant is added in the proper position on the file-system. Each variant or version of a document is entirely stored on the IsaWiki server in a specific data format, associated with some meta-information and a unique version identifier. 
The IsaWiki client is able to request any version/variant of any document in any data format, through a specific URL. For instance, the version 12.1 of the local document index.html in DOC format can be obtained getting the page http: //serverIsaWiki/index.doc_12.1; similarly http://serverisaWiki/index.html_12.2 returns the following version of the same document in HTML format. As expected, all the internal data conversions are performed according to the superior standard model described in the section 3.3 .

In the figure 3, you can see a PDF document converted into HTML and re-flowed into a different layout:

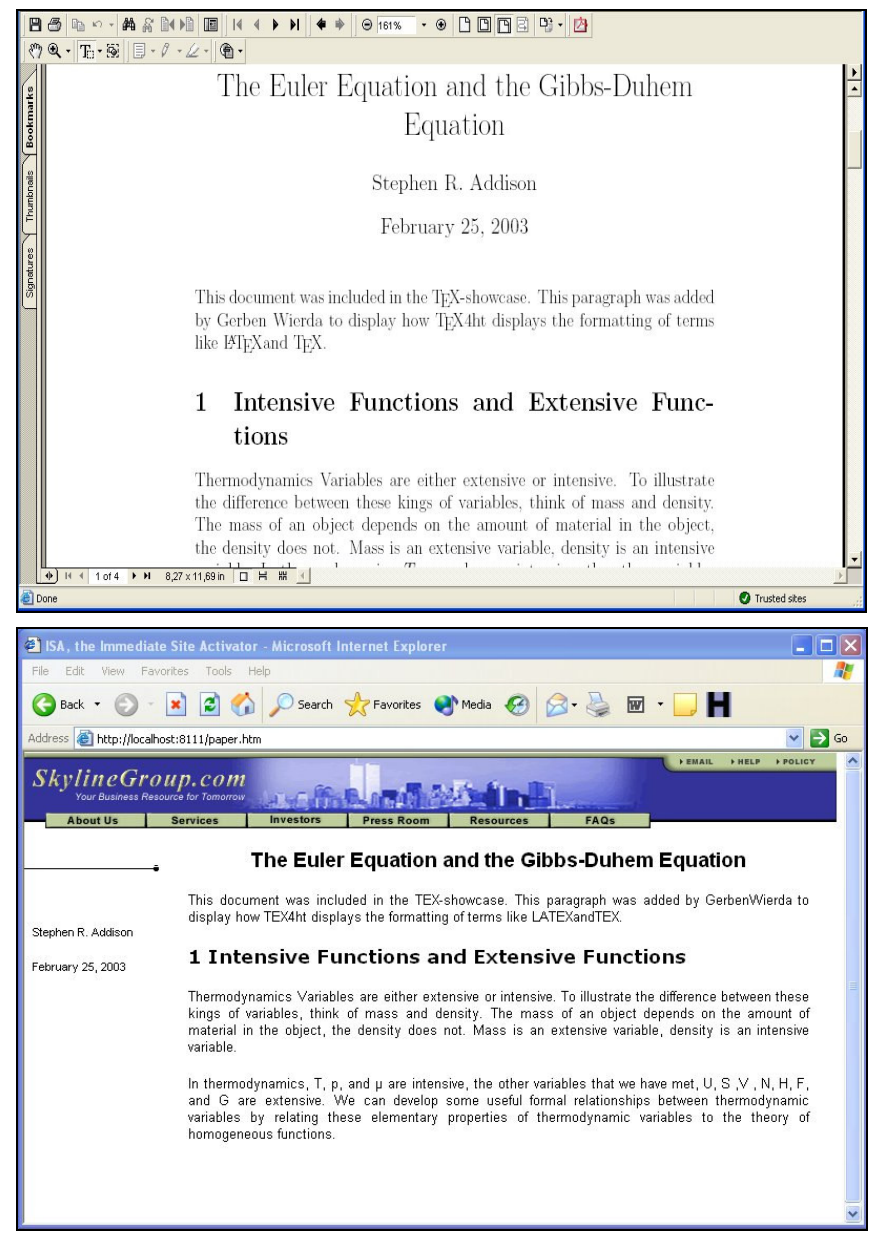

Figure 3. A PDF document re-flowed into a HTML page

Ideally any content could be re-flowed in any layout and any fragment can move from a page to another, according to the idea that a document is composed of individually manageable content assets and, separately, layout and style.

The templating engine of IsaWiki is directly imported from a previous project called ISA [35]. ISA is a web page production system based on the exploitation of standard desktop tools for the creation of content and layout and their automatic merging into the final web pages. In the scenario of producing a web site with ISA, a graphic designer creates the overall graphical aspect of the page using Fireworks or Photoshop. She/He will then use the slicing tool of the application to specify which area will contain which content. Independently, the content producer can proceed to write the content documents through MS Word, using styles as instructed by the layout designer. After the layout has been created and the content document written and saved on the server, ISA is able to merge them to form a complete web page.

Furthermore the IsaWiki server implements an advanced versioning and diff tool for XML documents allowing users to display differences between two versions (both of them have to be previously converted into the generic data format) or rebuild a multi-versions document in a single page, where all the contributions are clearly emphasized.

A number of services to handle local data are provided too. These include a simple search engine, a recent change document (where information about the most recently modified documents are listed), a configuration panel for the administrator of the server, a simple panel for users management and write/read permissions settings. Finally, advanced caching mechanisms are integrated in the server to improve efficiency.

Other functionalities are under development yet: firstly, a more advanced users' management that provides private working areas, users profiling and a fine-grained management of the fragments' ownership and intellectual propriety.

Having a full-featured users' management, the core of the IsaWiki system will be complete. In fact, we think that GE could be implemented integrating three main core elements: assets management, versioning and users' management. Their integration will allow users to create multi-sources documents (drawing content and ideas for all the accessed pages) or multiauthored documents (written in collaboration by many "emergent collaborators"), so that all the contributions will be clearly distinguishable. From this viewpoint, an advanced visualization of versioned documents is an essential requirement: in particular, the system should be able to display what has been changed from a version/variant to another one and what are the different contributions into a single document.

XanaWord [10], a previous work from which IsaWiki took inspiration, has already shown the potentiality and the feasibility of such a system. XanaWord is a web publishing environment for the customization just allowing users to edit web pages during the navigation. Whenever a user asks to edit the current page, an instance of MS Word is launched and loaded with the requested page; the user edits the content and saves it in a specialized server. The system will then extract the changes introduced in the editing session, and will create a personal variant (or a new official version) of the document. Anytime the user requests the same document the system will then add the changes and will provide the user with the modified resource, while the original copy remains still unchanged.

The XanaWord system is based on versioning, external anchoring and a non-transparent HTTP proxy to provide the required service. When a document is requested, all changes introduced by each user (extracted through a forward delta based versioning engine that exploits the versioning functionalities of MS Word) are applied on-the-fly to the original document retrieved from the origin server. Client-side, all versioning features can be requested by the user though an ad hoc menu integrated in Internet Explorer. Particularly interesting is the possibility of displaying a unique document, containing a version where all the previous changes are shown with different colors, as shown in the figure 4. 


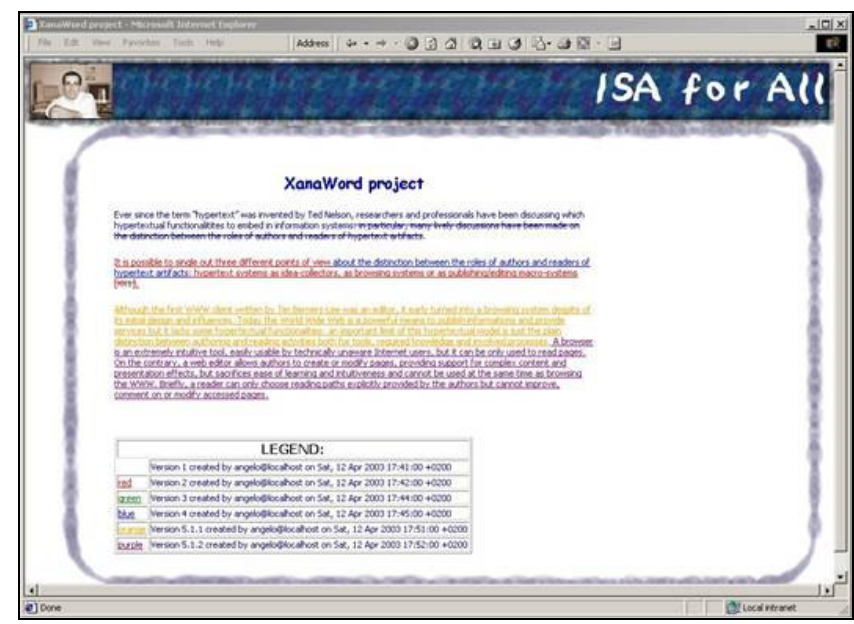

Figure 4. A XanaWord page showing multiple changes in a single document

In this case, each fragment belongs to a different version of the same page (probably created by the same author), but the same principle and engine can be adapted to control and display fragments into a multi-sources or a multi-authored document.

\section{CONCLUSIONS}

In this paper we re-propose an old vision of the World Wide Web as a sharable, universal and democratic environment where all users are entitled to modify, customize and re-use others' content. This model is just called "global editability" (GE). It has been directly inspired by Xanadu and by the original design of the same WWW, which initially was a wholly writable medium rather than a read-only one as today.

We have discussed about the advantages of a GE approach and about the IsaWiki system that really implements such a model. GE improves and integrated some existing web authoring approaches: the web data collection and reuse of any material, the possibility of annotating and linking external resources, the possibility of editing pages during the navigation and the collaborative editing.

IsaWiki is a distributed system, based on client-side architecture, designed and implemented according to the GE paradigm. Obviously a lot of technical and non-technical issues had to be solved designing such a system. This paper explains these problems and motivates the GE solution: the core principle is that any content fragment can be identified and retrieved within the system. Thus, it is always possible to know who edited a page, when edited and what was edited, so that any web user can modify any web page in a safe and controlled way.

Three main features could be integrated towards the implementation of such a global environment: documents' segmentation into manageable assets of content, advanced versioning and diff and users management. These functionalities are already implemented in IsaWiki and they will be soon integrated each other and strengthen.

In fact, the development of IsaWiki is not completed yet. We are working towards the creation of an advanced users' software manager, the integration of all the functionalities into a unique global environment, the support for new data formats, and the improvement of the client-side interface and so on.

\section{REFERENCES}

[1] Amaya, W3C, http://www.w3.org/Amaya/.

[2] Anderson K. M., Taylor R. N., and Whitehead E. J., "Chimera: Hypermedia for heterogeneous software development environments". ACM Transactions on Information Systems, 18(3), July 2000.

[3] Bieber M., Vitali F., Ashman H., Balasubramanian V., Oinas-Kukkonen H. (1997) "Fourth Generation Hypertext: Some Missing Links for the World Wide Web", International Journal of Human-Computer Studies, 47, 1997, 31-65.

[4] Blood , R. Weblogs: a history and perspective, http://www.rebeccablood.net/essays/weblog_history.html.

[5] Bouvin N. O. Unifying strategies for Web augmentation. Proceedings of ACM Hypertext'99, p 91-100, 1999.

[6] Carr L. A., DeRoure D., Hall W., and Hill G. "The distributed link service: A tool for publishers, authors and readers". Proceedings of the 4th International World Wide Web Conference, 1995.

[7] Catlin T., Bush P. and Yankelovich N., "InterNote: Extending a hypermedia framework to support annotative collaboration". In: Proceedings of Hypertext'89, (ACM Press, New York, 1989,) pp. 365-378.

[8] Ciancarini P., Folli F., Rossi D. and Vitali F. "XLinkProxy: external linkbases with XLink". In Proceedings of the 2002 ACM symposium on Document Engineering edited by ACM Press, 2002, p. 57-65.

[9] Cunningham, W. \& Leuf B. The Wiki way. New York, Addison-Wesley, 2001

[10] Di Iorio A., Vitali F. "A Xanalogical collaborative editing environment". In Proceedings of the second international workshop on Web document Analysis, University of Liverpool, (2003).

[11] Di Iorio A., Vitali F. "Web authoring: a closed case?". Accepted at Hawaii International Conference on System Sciences, January 3-6, 2005, Big Island (Hawaii). To be printed.

[12] Di Iorio A., Vitali F. "Writing the Web" in Journal of Digital Information, Volume 5, Issue 1, May 2004.

[13] Diaz L.M., Wustner E., Buxmann P. "Inter-organizational Document Exchange - Facing the Conversion Problem with XML", Proceedings of the ACM Symposium on Apllied Computing (SAC 2002), Madrid 2002

[14] Fetscherin, M. (2002): "Present State and Emerging Scenarios of Digital Rights Management Systems", in :The International Journal on Media Management, Volume 4, No. 3, 2002

[15] Grønbæk K., Sloth L., and Ørbæk P. "Webvise: browser and proxy support for open hypermedia structuring mechanisms of the World Wide Web". Proceedings of the 8th World Wide Web Conference, p 253-267, 1999.

[16] Gupta S., Kaiser G., Neistadt D., Grimm P., "DOM-based Content Extraction of HTML Documents", Proceedings. of WWW2003, May 20-24, 2003, Budapest, Hungary. 
[17] Haake A. and Hicks D. "VerSE: Towards Hypertext Versioning Styles" in Proceedings of ACM Hypertext '96, Washington DC, 224-234, March 1996.

[18] Hall W., Davis H. C., and Hutchings G. Rethinking Hypermedia: The MicroCosm Approach. Kluwer Academic, Norwell, USA, 1996.

[19] Iannella R., “Open Digital Rights Language”, W3C, http://www.w3.org/TR/odrl/, last visited $8^{\text {th }}$ November 2004.

[20] iMarkup: Annotate, organize and collaborate on the Web, http://www.imarkup.com/products/annotate_page.asp

[21] JotSpot Inc., "JotSpot Beta: the application wiki", http://www.jotspot.com/

[22] Kahan J., Koivunen M., Prud'Hommeaux E., and Swick R. "Annotea: An open RDF infrastructure for shared Web annotations". Proceedings of the WWW10, International Conference. Hong Kong, 2001.

[23] Laurillau Y. and, Nigay L., "Clover architecture for groupware" in the Proceedings of the 2002 ACM conference on Computer supported cooperative work, 2002, ACM Press, pp. 236-245.

[24] Maioli C., Sola S., and F.. "Wide-Area Distribution Issues In Hypertext Systems" in Proceedings of ACM SIGDOC '93, Kitchener, Canada, 185-197, 1993.

[25] Maioli C., Sola S., Vitali F., "The Support for Emergence of Collaboration in a Hypertext Document System" in: $A C M$ CSCW'94 Workshop on Collaborative Hypermedia Systems, Chapel Hill (NC), GMD Studien n. 239, ACM, 1994.

[26] Meyrowitz N., “Hypertext---does it reduce cholesterol, too?", IRIS Technical Report 89-9, Brown University, Institute for Research in Information and Scholarship, (November 1989). Transcript of Hypertext '89 keynote address.

[27] Miles-Board, T., Carr, L., Kampa, S. and Hall, W. (2003) "Supporting Management Reporting: A Writable Web Case Study". In Proceedings of The Twelfth International World Wide Web Conference (WWW2003), pages pp. 234-243, Budapest, Hungary.
[28] Nelson T.H., "Transcopyright: Dealing with the Dilemma of Digital Copyright." Educom Review, vol. 30, Jan/Feb 1997, p. 32 .

[29] Nelson T.H., "Managing immense storage". BYTE, Volume 13, Issue 1 , January 1988, pp. 225-238.

[30] Nelson T.H., Literary Machines. Sausalito (CA), USA, Mindful Press, 1987.

[31] Opt-in email marketing Resource Center, http://www.opt-inemail-marketing.org/, last visited $8^{\text {th }}$ November 2004.

[32] Raymond E.S, "The Cathedral \& the Bazaar (Hardback) Musings on Linux and Open Source by an Accidental Revolutionary", O'Reilly Editions, first edition October 1999.

[33] Schraefel, M. C., Zhu, Y., Modjeska, D., Wigdor, D. and Zhao, S. (2002) "Hunter Gatherer: Interaction support for the creation and management of within-web-page collections". In Proceedings of International World Wide Web Conference, pages pp. 172-181, Honolulu, Hawaii, USA.

[34] The International DOI Foundation, "The Digital Object Identifier", http://www.doi.org/, last visited $8^{\text {th }}$ November 2004.

[35] Vitali F., "Creating sophisticated web sites using well-known interfaces" in: HCI International 2003 Conference, Crete (Greece), June 2003.

[36] Vitali F., "Versioning Hypermedia", ACM Computing Surveys, 31(4), 1999, article n. 24, pp. 7.

[37] Vitali F., Di Iorio A., Ventura Campori E., "Rule-based Structural Analysis of Web Pages". In Simone Marinai and Andreas Dengel Eds., Document Analysis System VI, Volume 3163 of Lecture Notes in Computer Science, pp. 425-437, Springer Verlag, Berlin 2004.

[38] Vitali F., Durand D., "Using Versioning to Provide Collaboration on the WWW", The World Wide Web Journal, 1(1), 1995, 37-50.

[39] Wickens, C. D., Hollands, J.D. Engineering Psychology and Human Performance, 3rd Ed., Prentice Hall, 2000.

[40] Wiki Philosophy FAQ, WikiWikiWeb, http://www.c2.com/cgi/wiki?WikiPhilosophyFaq. 